\title{
Percutaneous aortic valve replacement has come to stay
}

\author{
Oscar A. Mendiz \\ Department of Interventional Cardiology, University Hospital Favaloro Foundation, Buenos Aires, Argentina
}

Percutaneous aortic valve replacement (TAVR) is a cutting-edge technology and one of the breakthroughs in cardiovascular medicine in the last decade and, for these reasons, some considerations must be taken even at risk that some of them might soon become outdated as a result of the exponentially growing medical evidence and experience about this technique [1].

The number of patients who could benefit from a TAVR is highly variable in different regions of the world, and many of them do not even have good estimations [2]. However, according to an European survey, approximately $30 \%$ of those patients with severe aortic stenosis requiring aortic valve replacement (AVR) do not currently receive the proper surgery for different reasons but high surgical risk appears as one of the most common cause for surgery contraindication [3]; moreover, considering that global population is aging, I have the expectation that the percentage and total number of patients who can benefit from TAVR will grow dramatically in few years.

These estimations can also vary depending on the threshold to indicate TAVR or AVR by open surgery (AVRbyOS) by each Heart Team. This will be influenced by recommendations in guidelines, local experience, and the economic situation of the region, which will affect cost/effectiveness relationship. For example, in Latin America, in those patients where TAVR may be an alternative with similar medical outcomes but more expensive, AVRbyOS will remain as the standard of care.

However, in some regions the target will not be this group of patients but rather those requiring AVR having a high risk or contraindication for AVRbyOS. In my opinion, octogenarians without other comorbidities will be the first intermediate risk group that will be electively treated by TAVR in a short time.

Patient selection must be performed by a multidisciplinary team with different training and expertise covering all the aspects that are necessary for taking care of this highly complex patient population, working in an adequate environment (Heart Team) [4, 5]. However, it is likely that these structures will simplify to make them more efficient, but access to consultations and specific care when required must be kept.

This also leads me to a belief that the practice will continue on an upward trend in the use of femoral approach, reserving all the other vascular access for particular situations. Nevertheless, vascular complication rate, which is an important predictor of mortality, should be maintained low and minimized if possible [6-8]. Technological developments leading to devices and delivery systems miniaturization with new altogether with better vascular closure devices will facilitate this trend and will make the so call "minimalist approach" to increase. This approach consists of using local anesthesia [9-11], percutaneous access $[12,13]$ and transthoracic ultrasound for the implantation.

The published outcomes with the two most studied devices worldwide are very encouraging despite the initial series have used first-generation devices and included the learning curve of the operators [14-17]. Although there are not randomized clinical trials comparing balloon expandable systems vs. self-expanding; there are large series of patients reported which did not show significant differences between them [18], except with valve-in-valve for the treatment of degenerated bioprosthesis, where there has been found a lower residual gradient using the CoreValve (Medtronic's self-expanding system) [19].

Address for correspondence: Dr Oscar A. Mendiz, Departamento de Cardiología Intervencionista, Hospital Universitario, Fundación Favaloro, (C1093AAS) Belgrano 1746 - 4 Piso, Ciudad Autónoma de Buenos Aires, Argentina, tel: (54 11) 4378-1359, fax: (54 11) 4378-1361, e-mail: omendiz@ffavaloro.org 
On the other hand, most of the series have shown an increased incidence of vascular complications using the Edwards system, mainly with the larger first generation device; and a higher need for permanent pacemaker implantation (PPM) when the CoreValve system is used [18]. The possibility of partial repositioning is a potential advantage of CoreValve, and possibility of recapturing and complete repositioning is a feature shared by other new devices that have been introduced to the market recently.

Postprocedural residual paravalvular leaks leaving a moderate-severe aortic regurgitation (AR) are associated with worse clinical outcomes; mainly with a higher 1-year mortality rate [20].

Proper planning, including patient selection using multiple images, taking into account the elliptical shape of the aortic annulus are extremely important not only for the patient but also for device selection [21]. Along with more experienced operators [22] and improvement in the design of the device, it will definitively reduce paravalvular leaks and other complications.

At the beginning of the study, the efforts to correct a moderate AR were not frequent, as many operators believed that it would be very well tolerated and will have no impact on late clinical evolution, but this concept has changed dramatically. However, if we do not get an optimal outcome after trying to correct all potential causes of leaks and $\mathrm{AR}$, we have to be very cautious when thinking about the possibility of an emergency AVRbyOS in this situation.

Conduction disturbances occur frequently after TAVR, being left branch blocking the most common. Although, in a series it was associated to high long-term mortality rate, this has been refuted by later series including more patients. Nor has an increase in late mortality due to the need for PPM [23], which is more frequent after TAVR than AVRbyOS. Although it has been a downward trend, it has been higher with CoreValve in comparison with Edwards-Sapien valves. A high implantation of the prosthesis ( $<6 \mathrm{~mm}$ from aortic annulus) and the absence or a conservative predilatation decrease the need for PPM, while the new onset left bundle branch block increases it [24]. PPM is not a determining factor for long-term outcomes, but ideally this requirement should be less.

Periprocedural stroke rate was a significant concern after the publications of the initial series reporting a stroke rate between $5 \%$ and $10 \%$, however this incidence has declined over time (3.8-6.7\% in more recent publications; $2 \%$ in our series). Although the stroke rate is lower than before, many efforts are done trying to improve these outcomes even more, but it does not seem an easy goal to be achieved. In the PARTNER trial, periprocedural stroke was more frequent in the group of patients receiving TAVR vs. those receiving AVRbyOS.

Cerebral embolization, demonstrated by new ischemic defects detected by magnetic resonance imaging is highly frequent but it has limited clinical manifestation [25]. This study has also shown that the size of the ischemic defects, when they occur, are bigger in the surgical group in comparison with TAVR patients [26].

Procedural improvements, such as decreasing the number of maneuvers over the aorta and calcified valve, less aggressive or no pre- and post-dilatation when they are not mandatory, would seem to be the cause of the decline of periprocedural stroke rate in later series [27, 28]. The uses of aortic flow deflectors and cerebral protection devices is an appealing concept which has been tested in some preliminary clinical studies which have not yet confirmed the effectiveness of these devices [29, 30].

Complete and easy valve repositioning is a common feature of many new devices which increase device manipulations over the aorta and calcified valve may also increase the potential of cerebral embolization and stroke risk. Nonetheless, this has been only a theoretical concern so far.

While TAVR was introduced for percutaneous replacement in patients with severe aortic stenosis and contraindications or high surgical risk, immediately after, came up the possibility to use it in cases of pure or predominant AR and also in cases of degenerated surgical bioprosthesis implanted by a prior open-heart surgery.

There are some recent series reporting promising outcomes with the use of CoreValve [31] and JenaValve [32] in patients with AR, with a higher need of intraprocedural valve-in-valve in the series with CoreValve. However, it would be ideal to have a randomized clinical trial before it is routinely used in daily clinical practice.

A different situation occurs in those patients requiring valve-in-valve due to degenerated surgical bioprostheses, because the information provided by the multicenter registry [33] can be considered enough, since the option of re-operation has a higher risks for most of these patients. Perhaps the only data to be confirmed is the long-term durability of the percutaneous graft in this specific situation where it has not been widely tested. 
Patients with thrombus or active endocarditis of the biological bioprosthesis are not candidates for this technique.

Long-term follow-up (beyond 10-15 years) is still a major limitation for widespread use of TAVR, but it is expected a similar durability to the surgical bioprosthesis. Moreover, valve-in-valve procedure for endovascular valves is also possible at follow-up and can extend valve durability ("secondary durability"), while many researches are conducted to find new synthetic compound that can replace biological tissue that may degenerate over the time.

The presence of concomitant mitral regurgitation increases the risk and uncertainty about late evolution and potential need for mitral valve repair or replacement after TAVR at follow-up. However, some recently published data from a substudy of the PARTNER Trial showed that a significant number of these patients have a substantial improvement in the degree of mitral regurgitation and these results have been better for those who received TAVR vs. those who only receive AVRbyOS without any intervention on the mitral valve.

The use of new percutaneous techniques to repair, and eventually to replace, mitral valve will add a complementary alternative to a higher risk population.

Dual adjunctive antiplatelet therapy with aspirin and clopidogrel has been used as the default strategy, but it has been a practical application. Nonetheless, this concept will be revisited since there are no studies to support this strategy over others [34]. Moreover, keeping in mind that many of these patients are elderly with high risk for bleeding, a more effective dual antiplatelet therapy strategy may be justified to decrease the stroke rate occurring after the procedure but it should be counterbalanced with a higher risk of bleeding in many of them.

Those patients who have concomitant atrial fibrillation, which is a predictor of stroke at 1 -year follow-up, and at high risk of bleeding, may benefit from left atrial appendage closure instead of receiving long-term oral anticoagulation [35].

Coronary revascularization prior to TAVR in those patients with severe coronary artery disease is a widely used approach for this comorbid situation, however, there is no solid evidence to justify this strategy in patients without previous angina or demonstrated ischemia [36]. We can consider it only an expert consensus, which may extrapolate some concept from cardiovascular surgery and the intention to simplify the percutaneous transluminal coronary angioplasty, which is technically more complex after TAVR. To perform both procedures in the same setting appears to be restricted to few cases, due to the frailty of many of them and the high risk of contrast-induced nephropathy.

We can conclude that although several issues still need to, and could be improved, TAVR is consolidating in daily practice as an excellent alternative to AVR by open-heart surgery. A larger clinical evidence provided by randomized clinical studies with long-term follow-up will conclude that TAVR is the first choice for high surgical risk patients and a good alternative for many others with intermediate risk who are still under discussion. We all agree on that we are witnessing a paradigm shift in the treatment of aortic stenosis with this new minimally invasive and highly effective transcatheter procedure that has come to stay.

\section{Conflict of interest: Medtronic (Proctor CoreValve)}

\section{References}

1. Olasińska-Wiśniewska A, Grygier M, Lesiak M, Trojnarska O, Grajek S. Transcatheter aortic valve implantation: The new option for high-risk patients with aortic stenosis. Cardiol J, 2011; 18: 461-468.

2. Nkomo VT, Gardin JM, Skelton TN et al. Burden of valvular heart diseases: a population-based study. Lancet, 2006; 368: 1005-1011.

3. Lung B1, Cachier A, Baron G et al. Decision-making in elderly patients with severe aortic stenosis: Why are so many denied surgery? Eur Heart J, 2005; 26: 2714-2720.

4. O'Sullivan CJ, Stortecky S, Buellesfeld L, Wenaweser P, Windecker S. Preinterventional screening of the TAVI patient: How to choose the suitable patient and the best procedure. Clin Res Cardiol, 2014; 103: 259-274.

5. Dubois C, Coosemans M, Rega F et al. Prospective evaluation of clinical outcomes in all-comer high-risk patients with aortic valve stenosis undergoing medical treatment, transcatheter or surgical aortic valve implantation following heart team assessment. Interact Cardiovasc Thorac Surg, 2013; 17: 492-500.

6. Généreux P, Webb JG, Svensson LG et al. PARTNER Trial Investigators. Vascular complications after transcatheter aortic valve replacement: Insights from the PARTNER (Placement of AoRTic TraNscathetER Valve) trial. J Am Coll Cardiol, 2012; 60: 1043-1052.

7. Reidy C, Sophocles A, Ramakrishna $\mathrm{H}$ et al. Challenges after the first decade of transcatheter aortic valve replacement: Focus on vascular complications, stroke, and paravalvular leak. J Cardiothorac Vasc Anesth, 2013; 27: 184-189.

8. Stępińska J, Czerwińska K, Witkowski A et al. Risk factors for bleeding complications in patients undergoing transcatheter aortic valve implantation (TAVI). Cardiol J, 2013; 20: 125-133.

9. Greif M, Lange $\mathrm{P}$, Näbauer $\mathrm{M}$ et al. Transcutaneous aortic valve replacement with the Edwards SAPIEN XT and Medtronic CoreValve prosthesis under fluoroscopic guidance and local anesthesia only. Heart, 2014; 100: 691-695.

10. Ruggeri L, Gerli C, Franco A et al. Anesthetic management for percutaneous aortic valve implantation: An overview of world- 
wide experiences. HSR Proc Intensive Care Cardiovasc Anesth, 2012; 4: 40-46.

11. Yamamoto M, Meguro K, Mouillet G et al. Effect of local anesthetic management with conscious sedation in patients undergoing transcatheter aortic valve implantation. Am J Cardiol, 2013; 111: 94-99.

12. Griese DP, Reents W, Diegeler A, Kerber S, Babin-Ebell J. Simple, effective and safe vascular access site closure with the double-ProGlide preclose technique in 162 patients receiving transfemoral transcatheter aortic valve implantation. Catheter Cardiovasc Interv, 2013; 82: E734-E741.

13. Vavuranakis M, Kariori M, Voudris V et al. Predictive factors of vascular complications after transcatheter aortic valve implantation in patients treated with a default percutaneous strategy. Cardiovasc Ther, 2013; 31: e46-e54.

14. Leon MB, Smith CR, Mack M et al.; PARTNER Trial Investigators. Transcatheter aortic-valve implantation for aortic stenosis in patients who cannot undergo surgery. N Engl J Med, 2010; 363: 1597-1607.

15. Smith CR, Leon MB, Mack MJ et al.; PARTNER Trial Investigators. Transcatheter versus surgical aortic-valve replacement in high-risk patients. N Engl J Med, 364: 2187-2198.

16. Grube E, Laborde JC, Zickmann B et al. First report on a human percutaneous transluminal implantation of a self-expanding valve prosthesis for interventional treatment of aortic valve stenosis. Catheter Cardiovasc Interv, 2005; 66: 465-469.

17. Grube E, Schuler G, Buellesfeld L et al. Percutaneous aortic valve replacement for severe aortic stenosis in high-risk patients using the second- and current third-generation self-expanding CoreValve prosthesis. J Am Coll Cardiol, 2007; 50: 69-76.

18. Chieffo A, Buchanan G, Van Mieghem N et al. Transcatheter aortic valve implantation with the Edwards SAPIEN versus the Medtronic CoreValve revalving system devices. J Am Coll Cardiol, 2013; 61: 830-836.

19. Linke A1, Woitek F, Merx MW el al. Valve-in-valve implantation of Medtronic CoreValve prosthesis in patients with failing bioprosthetic aortic valves. Circ Cardiovasc Interv, 2012; 5: 689-697.

20. Hayashida K1, Lefèvre T, Chevalier B et al. Impact of post-procedural aortic regurgitation on mortality alter trancatheter aortic valve implantation. J Am Coll Interv, 2012; 5: 1247-1256.

21. Watanabe Y1, Morice MC, Bouvier E et al. Automated 3-dimensional aortic annular assessment by multidetector computed tomography in transcatheter aortic valve implantation. J Am Coll Cardiol Intv, 2013; 6: 955-964.

22. Van Mieghem NM, Chieffo A, Dumonteil N et al. Trends in outcome after transfemoral transcatheter aortic valve implantation. Am Heart J, 2013; 165: 183-192.

23. Buellesfeld L, Stortecky S, Heg D et al. Impact of permanent pacemaker implantation on clinical outcome among patients under- going transcatheter aortic valve implantation. J Am Coll Cardiol, 2012; 60: 493-501.

24. Piazza N, Nuis RJ, Tzikas A et al. Persistent conduction abnormalities and requirements for pacemaking six months after transcatheter aortic valve implantation. Eurointervention, 2010; 6: 475-484.

25. Ghanem A, Kocurek J, Sinning JM et al. Cognitive trajectory after transcatheter aortic valve implantation. Circ Cardiovasc Interv, 2013; 6: 615-624.

26. Kahlert P, Knipp SC, Schlamann M et al. Silent and apparent cerebral ischemia after percutaneous transfemoral aortic valve implantation: A diffusion-weighted magnetic resonance imaging study Circulation, 2010; 121: 870-878.

27. Mendiz OA, Fraguas H, Lev GA et al. Transcatheter aortic valve implantation without balloon predilation: A single-center pilot experience. Catheter Cardiovasc Interv, 2013; 82: 292-297.

28. Grube E, Naber C, Abizaid A et al. Feasibility of transcatheter aortic valve implantation without balloon pre-dilation: A pilot study. J Am Coll Cardiol Cardiovasc Interv, 2011; 4: 751-757.

29. Onsea K, Agostoni P, Samim M et al. First-in-man experience with a new embolic deflection device in transcatheter aortic valve interventions. EuroIntervention, 2012; 8: 51-56.

30. Naber CK1, Ghanem A, Abizaid AA et al. First-in-man use of a novel embolic protection device for patients undergoing transcatheter aortic valve implantation. EuroIntervention, 2012; 8: 43-50.

31. Seiffert M, Diemert P, Koschyk D et al. Transcatheter aortic valve implantation for pure severe native aortic valve regurgitation. J Am Coll Cardiol, 2013; 61: 1577-1584.

32. Seiffert M1, Diemert P, Koschyk D et al. Transapical implantation of a second-generation transcatheter heart valve in patients with noncalcified aortic regurgitation. J Am Coll Cardiol Cardiovasc Interv, 2013; 6: 590-597.

33. Linke A1, Woitek F, Merx MW et al. Valve-in-valve implantation of Medtronic CoreValve prosthesis in patients with failing bioprosthetic aortic valves. Circ Cardiovasc Interv, 2012; 5: 689-697.

34. Durand E, Blanchard D, Chassaing S et al. Comparison of two antiplatelet therapy strategies in patients undergoing transcatheter aortic valve implantation. Am J Cardiol 2014; 113: 355-360.

35. Chun KR1, Bordignon S, Urban V et al. Percutaneous left atrial appendage closure with the AMPLATZER cardiac plug device in patients with nonvalvular atrial fibrillation and contraindication to anticoagulation therapy. J Am Coll Cardiol, 2013; 62: 96-102.

36. Van Mieghem NM, van der Boon RM, Faqiri E et al. Complete revascularization is not a prerequisite for success in current transcatheter aortic valve implantation practice. J Am Coll Cardiol Cardiovasc Interv, 2013; 6: 867-875. 